\title{
O Brasil e a América do Sul: (re)pensando a segurança e a defesa na região
}

\author{
Brazil and South America: \\ (re)thinking security \\ and defense in the region
}

Rev. Bra. Est. Def. ano 1, no 1, jul./dez., p. 112-146

ALEXANDRE FUCCILLE*

Tradução para o inglês de Laura Lammerhirt

\section{INTRODUÇÃO}

Falar de Brasil no contexto sul-americano implica, necessariamente, falar em números grandiosos: $48 \%$ da área total do subcontinente, $50 \%$ de sua população, $59 \%$ de sua economia/PIB (detendo cerca de $75 \%$ da base industrial e $2 / 3$ de sua agricultura), $42 \%$ da produção de petróleo, quase $3 / 4$ dos desembolsos voltados à infraestrutura da integração regional, entre outros indicadores igualmente superlativos. Tais cifras certamente impactam e obrigam-nos a olhar com mais vagar a questão da permanência e mudança na arquitetura de segurança e defesa do subcontinente na presente época.

O século XXI inaugura um novo período no relacionamento entre os países da América do Sul e, em particular, do Brasil para com estes. A erosão da influência norte-americana na região (informada por outras prioridades), ao lado de um maior protagonismo brasileiro e um sistema internacional com novos vértices de poder - a despeito da manutenção da unipoloaridade -, afetaram decisivamente a forma pela qual a segurança e defesa no subcontinente eram pensadas e conduzidas há mais de meio século. Fundamentalmente, percebe-se um esforço que pretende representar

* Professor da UNESP (Universidade Estadual Paulista) e membro do Grupo de Estudos de Defesa e Segurança Internacional (GEDES). Doutor em Ciência Política pela UNICAMP, foi vencedor do "II Concurso de Teses sobre Defesa Nacional" (CNPq/MD) com a melhor Tese de Doutorado do biênio 2005/2006 e tem vários artigos publicados na área de segurança e defesa. E-mail: fuccille@hotmail.com. 
uma passagem de um arranjo caduco de segurança coletiva em direção a um modelo mais dinâmico de segurança cooperativa, contudo de contornos ainda incertos e cujo resultado final segue em aberto.

Posto isso, o propósito deste texto é procurar avaliar desde uma perspectiva brasileira a dinâmica, os avanços e os desafios nas amplas áreas de segurança e defesa no subcontinente no presente século, cotejados com movimentos como o giro à esquerda e centro-esquerda nos governos da região, o novo protagonismo externo brasileiro neste espaço geográfico, os avanços na integração regional e seus impactos (UNASUL, Conselho de Defesa Sul-Americano etc), culminando na constituição do que tem sido chamado por alguns analistas de um vigoroso Complexo Regional de Segurança (CRS).

Ao debruçarmo-nos sobre a bibliografia do tema aqui proposto, notamos que a mesma é caracterizada por uma constante ambivalência, ora sinalizando para uma cooperação salutar e sem volta entre os países sul-americanos, ora apontando para divergências de fundo insuperáveis, ao menos nos curto e médio prazos, e um papel ainda incerto por parte do Brasil neste processo.

\section{A "PRIMAVERA SUL-AMERICANA"}

Como resposta à deterioração socioeconômica assistida ao longo da década de 1990, que arrastou dezenas de milhões de pessoas à pobreza em uma região já marcada por imensas desigualdades de todo o tipo - fruto da adesão quase que incondicional aos preceitos do "Consenso de Washington” -, somado a um quadro político extremamente complexo e sensível caracterizado por uma paulatina corrosão das instituições, a passagem do milênio sinalizou com uma nova alvorada onde, distintamente da cena anterior, novos governos crescentemente identificados com as demandas da cidadania e dos movimentos sociais experimentavam uma oportunidade de condução dos negócios nacionais.

Simbolizada pela posse de Hugo Chávez Frías no governo da Venezuela em 1999, esta espécie de "primavera sul-americana” seria seguida pela assunção de novos governantes de esquerda e centro-esquerda ao longo de toda a primeira década do novo século, como Luiz Inácio Lula da Silva no Brasil (2003), Néstor Kirchner na Argentina (2003), Tabaré Vázquez no Uruguai (2005), Evo Morales na Bolívia (2006), Rafael Correa no Equador (2007) e Fernando Lugo no Paraguai (2008), que abririam as portas - em 
princípio - a novas possibilidades e formas de cooperação neste inédito contexto.

A grande contradição é que, ao contrário do que o senso comum parece sugerir, a emergência destas novas lideranças, acompanhadas de intensa mobilização popular e seu séquito de anteriormente excluídos do processo político, acabou por levar a uma nova situação onde,

As bases sociais dessas novas lideranças tendem a considerar que boa parte de suas dificuldades econômicas e sociais são consequências dos impactos vindos de seu exterior, passando a demandar dos governantes posicionamentos internacionais mais autônomos. Para não perder apoio político doméstico, essas lideranças por vezes exacerbam seus discursos e práticas de política externa. A consequência é que, paradoxalmente, a nova onda de líderes "esquerdistas" sul-americana, a qual se supunha ser facilitadora de uma futura pauta de política externa de integração regional por proximidade de identidades ideológicas, acaba por operar contra essa tendência. ${ }^{1}$

Poucas não foram as ocasiões em que as disputas no plano sub-regional, seja por intermédio da Comunidade Andina de Nações (CAN), do Mercado Comum do Sul (Mercosul) ou ainda da Aliança Bolivariana para as Américas (ALBA), fizeram dissipar energia que poderia ser canalizada conjuntamente e explicitaram as pesadas contendas de bastidores que orientam as chancelarias nacionais. ${ }^{2}$ Assim, o necessário salto epistemológico da governabilidade à governança seria, uma vez mais, ofuscado.

Não obstante, as históricas hipóteses de guerra e de conflito já eram coisas do passado. $\mathrm{O}$ incremento do preparo orientado em função de capacidades (não confundir com capabilities) ao invés das antigas ameaças, crescentemente é uma realidade. $\mathrm{O}$ fim da guerra fria, o novo ambiente democrático pós-regimes autoritários, a intensificação do processo de globalização e o que se convencionou chamar "novas ameaças" (normalmente via atores não-estatais), aumentou vertiginosamente a adoção das medidas de confiança mútua entre os países sul-americanos.

Por outro lado, a antiga antipatia que muitos dos novos presidentes do subcontinente nutriam por Washington, combinada à forma pouco habilidosa com que os interesses estadunidenses foram conduzidos por estas paragens, acabaram evidenciando a decrepitude crescente de instituições e mecanismos como a OEA, a Junta Interamericana de Defesa (JID), o TIAR, entre outros, dando sinais inequívocos de esgotamento do sistema 
de segurança hemisférico estruturado a partir das décadas de 1930-40.

O movimento recente de securitização do fenômeno do terrorismo na agenda política norte-americana acabou por gerar respostas contraditórias na América do Sul, quando não de aberta confrontação. A baixa prioridade estratégica historicamente conferida pelo Departamento de Estado yankee à região, ${ }^{3}$ combinada à profunda alteração da agenda internacional deste país pós-setembro de 2001, os sucessivos reveses em temas como implantação da Área de Livre Comércio das Américas (ALCA), os fracassos nas tentativas de eleger seus candidatos a secretário-geral da OEA, as negativas do Chile e México (então membros-rotativos do Conselho de Segurança da ONU) em dar seu aval à invasão do Iraque em 2003, entre outras questões, acabaram por consolidar um imaginário de espaço geográfico possível e passível de desenvolver políticas mais independentes, advindas de uma maior margem de autonomia. Ademais, a saída do estado de hibernação em que ficou o urso russo ao longo dos anos 1990 e o espetacular crescimento chinês e seu desembarque no subcontinente ajudaram a tornar ainda mais complexo este novo quadro.

De toda sorte, o fato para o qual estamos querendo aqui chamar a atenção é que, a despeito de disputas (pontuais ou não) entre os diferentes Estados sul-americanos no processo de construção e condução da integração regional, parece haver um fio condutor comum (excetuado a Colômbia) com respeito à temática segurança e defesa, que é a aspiração por parte das lideranças políticas do fim da heteronomia e ingerência norte-americana na região. Neste particular, a América do Sul, concretamente, parece vivenciar uma nova realidade, com o florescimento, inclusive, de novas institucionalidades, como o Conselho de Defesa Sul-Americano e outros mecanismos, não obstante uma preocupante aproximação militar entre Paraguai e Estados Unidos, notadamente após a suspensão do primeiro do Mercosul e da UNASUL em meados de 2012. ${ }^{4}$

Todavia, apesar de importantes transformações operadas no panorama sul-americano, este novo quadro não representou per si o rompimento ou a renúncia da lógica do imperativo das “pequenas soberanias”, tão necessário à criação dos arranjos transnacionais que reiteradamente aparecem como um anseio nos discursos dos diversos líderes locais e que, vale lembrar, são buscados ao menos desde o Congresso do Panamá, em 1826, idealizado por Simón Bolívar. As diferentes visões, por vezes conflitantes, sobre o que é integração e como ela deve ocorrer (nos mais distintos campos) continuam a predominar. 


\section{O BRASIL NO NOVO CONTEXTO}

A posse do presidente Luiz Inácio Lula da Silva em janeiro de 2003 traria importantes mudanças não só internas como também externas, dadas a estatura de maior player regional e as dimensões físicas, políticas, econômicas e militares que o Brasil possui. ${ }^{5}$ Recordemos que uma maior assertividade com relação à cooperação e integração sul-americanas, ainda que não constituam propriamente uma novidade quando olhamos retrospectivamente a história, recentemente ganha novos contornos a partir do esforço - da perspectiva brasileira - iniciado com o presidente Fernando Henrique Cardoso, aprofundado pelo presidente Lula da Silva (ambos dirigentes lastreados em uma forte diplomacia presidencial) e novamente reiterado pela atual presidente Dilma Rousseff.

No presente século, para além da integração física e econômica pretendida, o Brasil tem trabalhado na América do Sul procurando fomentar uma agenda comum de segurança e contra-arrestar a influência norte-americana na região (originalmente centrada na expansão de bases militares no subcontinente, reativação da IV Frota Naval e combate ao terrorismo e às drogas). A pactuação desta agenda comum permitiria, em tese, a criação até mesmo de uma comunidade de segurança, dada a centralidade da territorialidade na dinâmica dos estudos de segurança. Contudo, seja no nível regional ou ainda no sub-regional, obstáculos têm aparecido.

A marcha da integração tem seguido com avanços, impasses e por vezes retrocessos, desde a I Cúpula Sul-Americana, realizada em agosto/setembro de 2000 em Brasília, com os chefes de Estado da região. Se geografia é destino, como se diz na geopolítica, o Brasil e seus vizinhos pareciam estar despertando de um longo período de letargia e inação, ciosos da necessidade de pactuação de uma agenda comum de oportunidades e desafios. Como forma de escapar às armadilhas muitas vezes colocadas pela frágil institucionalização da democracia e instituições na região, arroubos populistas e/ou disputas por liderança no processo de integração sul-americano, o Brasil optou por apostar em um tipo de amálgama, a partir de Cardoso, mantendo o regionalismo aberto anterior e alicerçado na integração física (por meio da Iniciativa para a Integração da Infra-Estrutura Regional Sul-Americana/IIRSA), tendo, por finalidade, a promoção do desenvolvimento da infraestrutura de transporte, energia e comunicações. ${ }^{6}$ Destacando os progressos da perspectiva brasileira, por ora poderíamos listar como principais a estruturação da Comunidade Sul-Americana de Nações/CASA 
(posteriormente renomeada União de Nações Sul-Americanas/UNASUL), a criação do Conselho de Defesa Sul-Americano (em aberta oposição aos EUA, que procurou valer-se da Colômbia para bloquear esta iniciativa do Brasil) e o ingresso da Venezuela como membro pleno do Mercosul (a Bolívia já assinou o Protocolo de Adesão e as tratativas com o Equador têm avançado positivamente).

O Conselho de Defesa Sul-Americano é a proposta mais ousada no âmbito da temática aqui abordada, em que pese não explicitar defesa contra quem ou o quê. ${ }^{7}$ É importante assinalar que entre a apresentação da proposta, iniciada em conversas bilaterais de bastidores ao longo de 2006-2007, e a exposição pública e aprovação do CDS em dezembro de 2008, diversas foram as articulações para superar as diferenças de forma e conteúdo entre seus 12 membros, bem como sepultar a ideia de que tal instrumento visava consolidar a hegemonia brasileira na região. ${ }^{8}$ Entre uma Colômbia que se enxergava insulada, cercada de governos esquerdistas (para não falarmos dos estreitos vínculos político-ideológicos com Washington e a colaboração na área castrense via Plan Colombia), e uma Venezuela histriônica que defendia a criação de um instrumento militar operacional comum chamado Organização do Tratado do Atlântico Sul (OTAS, nos moldes da Organização do Tratado do Atlântico Norte/OTAN), ${ }^{9}$ acabou vingando a proposta de implementação de uma estrutura suavizada - subordinada a um bloco regional multipropósito como a UNASUL -, de concertação entre seus integrantes, capaz de fomentar o intercâmbio nos campos da segurança e defesa, cujas decisões só têm validade se acordadas por consenso e com previsão de ao menos um encontro anual ordinário entre seus ministros da Defesa. ${ }^{10}$

Para alguns atores deste processo, a acelerar a conformação deste arranjo (particularmente da perspectiva brasileira) estavam: 1) a possibilidade de transbordamento de "novas ameaças" a outros países, como o narcotráfico colombiano para o Brasil e a contenda envolvendo Colômbia, Equador e Venezuela pela morte de Raúl Reyes (número dois na hierarquia das Forças Armadas Revolucionárias da Colômbia-Exército do Povo/FARC-EP); 2) os conflitos sub-regionais como os contenciosos, envolvendo Peru e Equador, Bolívia e Chile, entre outros; 3) os movimentos autóctones difundindo um sentimento separatista, como ilustram Bolívia e Paraguai; 4) frear os ímpetos bolivarianos na região, sobretudo via isolamento da Venezuela e/ ou dos países integrantes da ALBA; 5) evitar uma corrida armamentista na região, com a adoção cresceste de medidas de confiança mútua (CBMs, do 
original Confidence Building Measures); 6) solidificar o Atlântico Sul como um área de paz, vital e livre de armas nucleares, e de projeção de poder ao Brasil, não permitindo a presença de potências extra-regionais (como o caso do Reino Unido e a questão Malvinas/Falklands); 7) impedir a materialização de uma política estratégica de segurança hemisférica definida a partir dos Estados Unidos para o subcontinente; e, último mas não menos importante, 8) concretizar os objetivos estratégicos da Política Externa Brasileira de consolidação de um processo de integração sul-americano. ${ }^{11}$

Efetivamente, mais do que nunca, para que o desenvolvimento do Brasil se acelere e se consolide sua pretensão de player global, a estabilidade joga um papel fundamental não apenas para o florescimento pleno de suas potencialidades, mas, igualmente, para mostrar ao mundo seu desempenho como fiador de uma importante estabilidade regional. ${ }^{12} \mathrm{O}$ comando da Missão de Estabilização das Nações Unidas no Haiti (MINUSTAH) desde 2004 não tem outra razão para além das justificativas protocolares de ajuda humanitária etc, constituindo-se em um verdadeiro ponto de inflexão no modus operandi brasileiro em operações de paz da ONU. ${ }^{13}$

Ao mesmo tempo, como frisa bem Alsina Jr. (2009, 181), "a satisfação territorial e a não securitização de ameaças emanadas dos países lindeiros permitem que o País [Brasil] priorize a dimensão do desenvolvimento em detrimento da dimensão estratégico-militar". Todavia, se historicamente foi assim, um país com mais de 15.000 quilômetros de fronteiras secas, cerca de 8.000 quilômetros de litoral e um imenso espaço aéreo, ${ }^{14}$ que reivindica um assento permanente em uma possível reconfiguração do Conselho de Segurança da ONU e pretende-se avalista da concórdia em seu entorno, não pode prescindir de uma estrutura militar crível e descurar de seus meios de defesa.

Em várias circunstâncias o Brasil não tem sido feliz, para dizer o mínimo, em erigir um discurso coerente e que pareça crível aos demais parceiros regionais, tendo como consequência um perfil identitário ainda não claramente delineado. Ao falar sobre o subcontinente e a relação do Brasil para com este, enquanto o presidente FHC destacava que "só teremos êxito se formos capazes de ser companheiros dos outros países. Não podemos sufocar, tirar vantagem desnecessária. A vantagem é estratégica, não é para o dia de amanhã”, por vezes temos afirmações como a do presidente Lula de que "é impressionante como todos esses países estão quase a exigir que o Brasil lidere a América do Sul”. ${ }^{15}$ Em que pese os esforços envidados por Lula da Silva para que a integração regional saísse do papel e a ação que o 
BNDES (Banco Nacional de Desenvolvimento Econômico e Social) passou a jogar para concretizar a IIRSA como um dos braços da política externa brasileira, em larga medida há um sentimento generalizado do Brasil como um hegêmona, gigante e subimperialista, permeando corações e mentes de boa parte dos vizinhos sul-americanos. ${ }^{16}$ Para que tal percepção se esvaia, é preciso que se avance, reduzindo efetivamente as importantes assimetrias que ainda caracterizam os países da região e apostando na integração regional para valer (e não apenas como mecanismo de salvaguarda a ameaças externas comuns de diferentes tipos e/ou um tipo de soft balancing). Não só os vizinhos menores, mas também o Brasil seria enorme beneficiário se tal processo ocorresse, dissipando definitivamente a imagem de um "gigante egoísta" - na feliz expressão de Andres Oppenheimer.

A dificuldade da presidente Dilma atualmente reside em um resiliente quadro externo de crise, que impacta diretamente os planos doméstico, regional e global (limitando a capacidade de ação do Estado brasileiro no sistema internacional), combinado ao pouco entusiasmo que a mandatária nutre pelas questões internacionais. Por outro lado, a crescente probabilidade de convulsão da Argentina, que se avizinha, somada a uma Venezuela fragilizada tanto econômica quanto politicamente, abrem novas portas para o Brasil transformar esta crise em oportunidade.

Assim, a construção de uma ativa e altiva comunidade de segurança com vistas à estabilidade regional aparece como tema e tarefa central nos marcos mais amplos desse processo, uma vez que, mesmo com a participação brasileira buscando dirimir desconfianças, criar entendimentos e facilitar o diálogo, a tão propalada identidade sul-americana em matérias de defesa até este momento é uma promessa não realizada. Porém, as políticas integracionistas avançam em diferentes velocidades, por vezes de forma mais acelerada no campo político - vide o Conselho de Defesa Sul-Americano -, todavia seguidas de um descompasso no campo econômico e outros, bem como de uma arquitetura institucional mais sólida, capaz de dar suporte a uma integração em redes mais profunda e profícua.

Ainda: o Brasil precisa capitanear este processo, sem prejuízo de suas parcerias estratégicas, deixando de exercer uma liderança envergonhada e regulamentando os ganhos - sendo desejável que isto aconteça de uma forma consorciada. Acreditamos que seja o momento de se superar o estéril debate acerca de qual a visão brasileira sobre a integração sul-americana - se como um fim em si mesmo ou plataforma de sua inserção global, haja vista que não há incompatibilidade, mas antes complementaridade entre 
os dois possíveis caminhos na minimização de fragilidades e maximização de sinergias. Já é passada a hora de ultrapassar as hesitações, embaraços e perplexidades dos últimos anos que, em verdade, apenas têm terrificado e paralisado muitas das iniciativas integracionistas.

\section{AS DIFICULDADES NA CONSTRUÇÃO DE UMA NOVA CULTURA ESTRATÉGICA}

A construção de uma nova cultura estratégica, não obstante a aproximação política e o incremento dos fluxos de comércio intra-regional que se seguiu ao fim dos regimes militares na região e o término da guerra fria, não é tarefa das mais fáceis. Foram séculos com os vizinhos se percebendo como as principais ameaças e orientando o preparo de suas Forças Armadas para a guerra com seus países lindeiros e/ou de seu entorno.

As considerações anteriores e a discussão em torno de uma nova cultura estratégica necessariamente nos levam a algumas questões: houve alteração importante na distribuição global de poder no sistema internacional nos últimos anos? Como essa repartição de poder afeta as opções sul-americanas e, em particular, as brasileiras? Temos instituições regionais fortes? O mundo político/civil dirige o mundo das armas/militar? Estas e outras questões impactam centralmente a reflexão que aqui estamos desenvolvendo.

Um primeiro elemento a ser destacado é a mudança relacional na posição dos Estados Unidos no sistema internacional. Os ataques de $11 \mathrm{de}$ setembro de 2001, pioneiros em grande proporção na porção continental norte-americana desde sua ascensão à condição de potência, não só abalaram o sentimento de incolumidade contemporâneo por parte daquela população como lançou seu sistema político em uma cruzada internacional nominada war on terror. A crise financeira iniciada em 2008, cujo epicentro se deu neste país, igualmente implicou em um declínio - ainda que relativo - da superpotência no conjunto das interações sociais que se desenvolvem na esfera internacional. Ainda assim, sem entrarmos em pormenores do renascimento russo a partir de Putin e do crescente protagonismo chinês, acreditamos que a polaridade do sistema internacional não foi alterada no período pós Guerra Fria, ${ }^{17}$ a despeito de novos vértices de poder que tem emergido no presente século, mas que ainda não conseguiram romper com a unipolaridade.

Ao lado disso, as urgências estratégicas e militares norte-americanas 
- notadamente Afeganistão e Iraque - possibilitaram como resultado concreto que, em uma região que ainda no período da Guerra Fria nunca esteve no topo das prioridades da grande potência, houvesse uma nova divisão de responsabilidades, onde o Brasil desponta como garantia da estabilidade sul-americana (inclusive procurando moderar os países bolivarianos), em uma espécie de liderança aquiescida, sem, contudo, confrontar severamente Washington no nível global.

Como pano de fundo, desde a formação do Mercosul em 1991 até o estabelecimento da UNASUL em 2008, temos visto um pulular de instâncias, processos e instituições que precisam ser fortalecidos, maturados e sedimentados. As céleres e constantes alterações e regressões de pontos anteriormente acordados entre os países do subcontinente pouco contribuem para o fortalecimento das instituições regionais - condição necessária, ainda que insuficiente - e de esteio para o aprofundamento das relações de segurança interestatais, que é o ponto que aqui mais de perto nos interessa. O exemplo mais dramático talvez seja dado pelo Mercosul, proposta de Mercado Comum, atualmente na fase de União Aduaneira (mais que imperfeita!), que tem levado importantes setores das sociedades de diferentes Estados integrantes deste bloco a defenderem que este arranjo retroaja a uma Área de Livre Comércio. Os reflexos de tal instabilidade podem ser sentidos, e.g., na não concretização de uma Agência de Segurança e Defesa do Mercosul, como previsto há mais de uma década, e cuja sede situar-se-ia em Montevidéu (a exemplo do Parlasul), dificultando a passagem e avanço do nível do diálogo bilateral para o diálogo subregional. ${ }^{18}$

Assim, no início da segunda década do novo século,

Os processos de integração permanecem, mas sem aprofundamentos. Na área de defesa e segurança atingiu-se um patamar significativo de mecanismos de confiança mútua, ao que parece satisfatório aos países. Ou seja, novos e ousados passos não são estabelecidos, ainda que com a criação da UNASUL e do Conselho de Defesa Sul-Americano. ${ }^{19}$

Junto a isto, sem desconsiderar muitas vezes a inépcia política civil e a lentidão/desconfiança das burocracias nacionais, vale destacar que os atores fardados não são meros coadjuvantes, e uma mentalidade tão arraigada e conservadora como a militar não se transmuta por uma simples troca de regime político e uma nova realidade histórica. Requer, em verdade, uma profunda alteração no plano cultural e dos signos socialmente construídos. 
Nessa direção,

As transformações culturais são naturalmente processos largos, não isentos de marchas e contramarchas. As mudanças nas concepções de segurança são parte desses processos, e na região têm estado marcadas por debates profundos acerca do que significa a segurança, qual é o papel das forças militares, e fundamentalmente, quais são as visões que cada país tem acerca do que desafia seu cenário de segurança. Isto causa impacto nas possibilidades multilaterais. ${ }^{20}$

Concomitantemente, o controle civil democrático sobre os militares deve ser um fato consolidado. Neste sentido, a direção política deve apontar para quais Forças Armadas são ambicionadas em função do perfil estratégico pretendido pelo país, que deverá contemplar o redimensionamento de seus meios, aqui incluso pessoal, material, educacional etc. Gostaríamos de assinalar que, em alguns países da região, o aparelho militar vem perigosamente assumindo tarefas que competem aos civis, com possíveis desdobramentos nada alvissareiros, para além da manutenção da já tradicional missão de fiadores (em diferentes graus) da ordem política e institucional que este preserva em muitas destas nações.

Uma nova cultura estratégica não surge entre o crepúsculo e a alvorada. De forma um tanto sumarizada e pensando em termos de região e acúmulo no tema em tela, vale listar a criação de mecanismos como o "Tratado Americano de Soluções Pacíficas"/Pacto de Bogotá (1948), a "Zona Livre de Armas Nucleares na América Latina"/Tratado de Tlatelolco (1967), a “Zona de Paz e Cooperação do Atlântico Sul” (1986), o "Acordo de Mendoza”, entre Argentina, Brasil e Chile (1991), proibindo a proliferação, a posse e o uso de armas químicas e biológicas, a "Zona de Paz Sul-Americana"/ Comunicado de Brasília (2000), a inauguração das "Reuniões de Ministros da Defesa da América do Sul” (a primeira ocorrendo no Rio de Janeiro em 2003), além do crescente adensamento das relações bilaterais entre Brasil e Argentina, desde a década de 1980, na área nuclear, com a abdicação da fabricação e emprego por ambos de armas atômicas e a instituição da Agência Brasileiro-Argentina de Contabilidade e Controle de Materiais Nucleares/ABACC (única organização binacional de salvaguardas nucleares do mundo), culminando no "Acordo Bilateral de Cooperação em Matéria de Defesa”, firmado em 2007. ${ }^{21}$ Enfim, um expressivo acervo e uma não desprezível tradição de se procurar distensionar o ambiente de segurança 
e defesa regional.

A partir de 2009, com o início efetivo de suas atividades, o Conselho de Defesa Sul-Americano tem aparecido como um importante espaço de concertação onde, a despeito das idiossincrasias regionais/ideológicas entre a CAN, o Mercosul, ou ainda a ALBA, importantes avanços têm sido registrados, como a definição de Planos de Ação comuns na temática de segurança e defesa, o estabelecimento de medidas de confiança mútua, a criação do Centro de Estudos Estratégicos de Defesa (CEED) na cidade de Buenos Aires, a construção de uma metodologia comum de medição dos gastos em defesa, um importante intercâmbio em matéria de formação e capacitação militar, a recém anunciada Escola de Defesa Sul-Americana, entre outros pontos igualmente meritórios. Não obstante, desafios permanecem.

\section{CONSIDERAÇÕES FINAIS}

Em face do exposto, a regionalização da arquitetura de segurança e defesa no subcontinente prossegue, mas com novas interrogantes: o que temos assistido, corrida armamentista ou reaparelhamento? E os históricos contenciosos fronteiriços e querelas territoriais, foram superados? Os espaços e identidades nacionais são respeitados reciprocamente? Como evitar desequilíbrios onde a economia/PIB - bem como os gastos na área de defesa - apenas do Brasil representam mais da metade de todos os outros países do subcontinente somados? Podemos - definitivamente - falar em superação da dicotomia Pacífico (arco de instabilidade/CAN) versus Atlântico (arco de estabilidade/Mercosul)? Como a questão amazônica se apresenta hoje? O risco de balcanização de países da região é coisa do passado? A tutela estratégica norte-americana foi contida?

Na verdade, o Conselho de Defesa Sul-Americano ainda não passou por nenhuma prova de fogo capaz de por em xeque a eficácia e a eficiência desta estrutura institucional, para além do gerenciamento (importante) de crises e a geração de externalidades positivas nos campos da segurança e defesa. Em paralelo, a chamada agenda negativa, que caracterizou o relacionamento EUA-América do Sul no período pós-Guerra Fria, centrada no combate ao tráfico de drogas, terrorismo e outros delitos de natureza policial, foi parcialmente superada. A despeito disso, os Subcomplexos Regionais de Segurança do Cone Sul e Norte-Andino vivenciam dinâmicas ainda bastante díspares entre si, que necessitam de maior articulação e organicidade. A integração sul-americana e a estruturação de um Complexo Regional de 
Segurança de tipo centrado têm avançado, de forma não desprezível, alicerçada muitas vezes em bases frágeis, como uma forte retórica nacionalista, defesa da soberania e não intervenção. ${ }^{22}$

O comportamento do Brasil, principal fiador da criação do CDS, carece de coerência e se apresenta inúmeras vezes de forma diacrônica. Se, por um lado, no mais alto nível político, Brasília trabalha pela concretização deste arranjo institucional (que já representa $2 / 3$ do destino de suas exportações em armamentos), por outro, descuida-se de engajar o próprio Ministério da Defesa e, mais particularmente, os militares brasileiros, a atuarem de forma mais decisiva e decidida pela concretização desta importante iniciativa e de seus mecanismos operativos. Na prática, vários altos oficiais e oficiais-generais veem com muita reserva este empreendimento, não poucas vezes classificando-o como uma "extravagância”, fruto de devaneios de um governo esquerdista, que não encontraria correspondência na realidade.

Se, iniludivelmente, a integração regional tem funcionado como uma estratégia para garantir mais voz (e força) aos pleitos da região em um mundo de crescentes assimetrias de poder, isto não pode eclipsar a absoluta fragilidade que tal instrumentalização deste importante processo representa para uma cooperação mais sistêmica, não obstante avanços pontuais. A passagem da segurança coletiva à segurança cooperativa é um fato. Enfim, a semente foi lançada, mas, para que esta viceje, será preciso muita atenção, cuidado e dedicação. O sentimento cultivado por parte da elite brasileira acerca de seu país como um "queridinho" na região é rarefeito em sua fundamentação. Se o Brasil de fato quiser liderar, é preciso vontade e mobilização para tanto, e que esteja ciente de que isto tem um preço (e não é barato!).

Ao longo de toda a primeira década do século XXI, as reuniões não só aumentaram de periodicidade, bem como novos arranjos institucionais foram pensados e implementados. Como resultado final, constatamos, neste breve balanço da dinâmica, avanços e desafios nos campos da segurança e defesa no espaço geográfico sul-americano, que o atual processo de integração é marcado por várias ambivalências, inconsistências, quando não abertas oposições. Mais: a construção de uma nova cultura estratégica, ainda que louvável, mostrar-se-á um "gigante de pés de barro" se não for acompanhada do respectivo aprofundamento e criação de novas institucionalidades nos planos econômico, social, cultural, entre outros. Neste sentido, esta contribuição, longe de pretender esgotar o tema em tela, propõe-se a ser uma reflexão em uma área ainda pouco explorada no subcontinente, somando-se às demais tentativas de interpretação do Brasil e da América do Sul contemporâneos e os papéis que estes almejam no presente século. 


\section{NOTAS}

1. Dupas \& Oliveira (2008), 239.

2. Apenas para ilustrar, isso pode ser facilmente verificado nos embates envolvendo a "diplomacia dos biocombustíveis" (Lula) versus a "diplomacia do petróleo" (Chávez).

3. Recentemente o secretário de Estado do governo Barack Obama, John Kerry, em discurso ante o Comitê de Assuntos Exteriores da Câmara de Representantes, classificou a América Latina como "quintal" dos Estados Unidos. Ver "John Kerry, Secretary of State: 'Latin America is our back yard" " (http://english. pravda.ru/world/americas/23-04-2013/124377-latam_backyard-0/; acesso em 04 de maio de 2013) e Colombo \& Frechero (2012).

4. Antes disso, exercícios militares por parte do Exército brasileiro na fronteira com o Paraguai em fins de 2008, em uma espécie de desagravo informal aos "brasiguaios" (principais produtores agrícolas naquele país) que sofriam pressão dos sem-terra local, mobilizaram um efetivo de milhares de homens, trazendo tensão e desconforto à região. Para piorar, desde 2010 a guerrilha também passou a ser uma nova e triste realidade neste sofrido país, por intermédio do Exército do Povo Paraguaio (EPP).

5. Nesse mesmo ano, teve lugar no Brasil, pela primeira vez na história, a " 1 a Reunião de Ministros da Defesa da América do Sul”. A Política Externa Brasileira ao longo do governo Lula (2003-2010) é motivo de muita controvérsia entre os analistas. Com respeito às questões de segurança e ao tratamento diferenciado que estas receberam ao longo dos dois mandatos do presidente Lula, sugerimos Villa e Viana (2010).

6. São mais de 500 projetos a um custo estimado de cerca de US\$ 120 bilhões. Para áreas de ação, projetos, planejamento e documentos da IIRSA, ver http:// www.iirsa.org/index.asp?CodIdioma=ESP (acesso em 15 mar. 2013).

7. Para uma cronologia da criação do CDS e as disputas envolvendo sua instituição, ver Medeiros Filho (2010), Carvalho (2009) e Teixeira (2011). Para um balanço mais amplo, cf. Pagliari (2009).

8. Um interessante panorama de como a inserção brasileira foi pensada desde a década de 1950 até os dias atuais pode ser conferido em Vigevani e Ramanzini Júnior (2010). A propósito da discussão em torno da ideia de liderança brasileira, vale a pena considerar Danese (2009).

9. Antes desta oportunidade, a última vez em que a criação de um mecanismo 
semelhante foi aventada deu-se nos anos 1980, no início do governo Ronald Reagan (1981-1989), onde Argentina e África do Sul eram entusiastas de tal proposta norte-americana. O Brasil e outros países africanos - notadamente os da chamada África ocidental - procuraram obstaculizar tal iniciativa. Já em meados de 1982, com a eclosão da Guerra das Malvinas, tal devaneio esboroava-se.

10. Acerca das suas funções e outras atribuições, ver o site http://www.cdsunasur. org/ (acesso em 27 mar. 2013).

11. Notas pessoais a partir de conversas off the record com membros do Ministério das Relações Exteriores (Itamaraty) e Ministério da Defesa brasileiros. 12. Para tanto, é fato que ao lado do tradicional soft power há inúmeros desafios colocados a fim de que o Brasil aumente seu hard power. Por exemplo, uma importante limitação advém do fato de este país ser o único membro do acrônimo BRIC desnuclearizado. Cf. Bertonha (2010) e Flores (2009-2010).

13. Para pormenores sobre a participação brasileira em Operações de Paz, ver Rezende (2012).

14. Corroborando estes elementos, a lembrança de que várias nações sulamericanas são menores geograficamente do que muitos Entes Federados brasileiros (e.g., se o Estado do Amazonas fosse um país seria o $3^{\circ}$ maior da América do Sul - depois de Brasil e Argentina), menos populosos (e.g., o Estado de Minas Gerais tem um população maior que Bolívia, Paraguai e Uruguai somados), de menor peso econômico (e.g., se o Estado de São Paulo fosse um país seria a $2^{\circ}$ maior economia do subcontinente, só perdendo para o próprio Brasil), entre outros pontos, não podem deixar de causar mal-estar entre seus vizinhos.

15. "Para América do Sul, liderança brasileira ainda é promessa" (http://www. bbc.co.uk/portuguese/reporterbbc/story/2008/03/080303_ams_abre1_diplomacia. shtml; acesso em 27 mar. 2013).

16. Em uma rápida busca pela internet isto é facilmente visualizável. Apenas para aclarar, ver Le Monde Diplomatique Brasil. Dossiê Imperialismo Brasileiro. Fevereiro de 2009; "O imperialismo brasileiro preocupa a região", O Estado de S. Paulo, 23/10/2008; "El imperialismo brasileño seguirá intacto con el gobierno de Dilma”, ABC Color (http://www.abc.com.py/articulos/el-imperialismo-brasilenoseguira-intacto-con-el-gobierno-de-dilma-178977.html; acesso em 27 fev. 2013); Samuel de Jesus. “ 'Imperialismo Brasileiro': visões jornalísticas sobre a atuação brasileira na América do Sul e no mundo" (http://mundorama.net/2012/09/25/ imperialismo-brasileiro-visoes-jornalisticas-sobre-a-atuacao-brasileira-na- 
america-do-sul-e-no-mundo-por-samuel-de-jesus/; acesso em 26 fev. 2013). Para um contraponto a estas leituras, cf. Curado (2011).

17. Em plena "crise", os Estados Unidos da América, que concentram menos de 5\% da população mundial, em 2012 continuavam responsáveis por $43 \%$ dos gastos militares e de defesa do planeta. Ver http://www.sipri.org/databases/milex (acesso em 12 jul. 2013).

18. Documentos pessoais da fase em que trabalhei na Secretaria de Política, Estratégia e Assuntos Internacionais do Ministério da Defesa no Brasil, de 2003 a 2005.

19. Grifo nosso. Cf. Soares (2011), p. 104.

20. Grifo nosso. Ver Donadio (2011), p. 115.

21. A esses poderíamos somar as iniciativas brasileiras dos Acordos BilateraisQuadro com Bolívia, Chile, Colômbia, Equador, Paraguai, Peru e Uruguai, além dos Acordos-Quadro de segurança regional assinados pelo Mercosul com Bolívia, Chile, Colômbia, Equador, Peru e Venezuela - todas deste século.

22. Para detalhes, a relação entre UNASUL e os referidos subcomplexos foi trabalhada por Fuccille e Rezende (2013). 


\section{INTRODUCTION}

Talking about Brazil in the South-American context necessarily implies talking about big numbers: $48 \%$ of the subcontinent's total area, $50 \%$ of its population, $59 \%$ of its economy/GDP (including about $75 \%$ of the industrial base and $2 / 3$ of its agriculture), $42 \%$ of oil production, almost $3 / 4$ of the expenditure on infrastructure of regional integration, amongst other equally superlative indicators. Such numbers certainly have an impact and oblige us to look closely to the question of permanency and change in the architecture of security and defense in the subcontinent nowadays.

The 21 st century inaugurates a new period in the relations between countries in South America, particularly the ones regarding Brazil. The erosion of North-American influence in the region (informed by other priorities), alongside a greater Brazilian protagonism and an international system with new vertexes of power - despite the maintenance of unipolarity -, hás decisively affected the way security and defense were thought and conducted more than half a century ago. Fundamentally, an effort that intends to represent the passage of an old-fashioned arrangement of collective security towards a more dynamic model of cooperative security is noticed, although its outlines are still uncertain and its final result is yet to be settled.

That being said, the purpose of this text is to evaluate the dynamics, from a Brazilian perspective, of the advances and challeges in the broad areas of security and defense in the sub-continent in the present century, in parallel to movements such as the rise of leftist and centre-leftist gouvernments in the region, the new external Brazilian protagonism in that geographic space, the advances of regional integration and its impacts (UNASUR, South-American Council of Defense, etc), reaching the peak with the constitution of what has been called by some analysts a vigorous Regional Security Complex (RSC).

Taking a look at the bibliography hereby presented, we noticed it is characterized by a constant ambivalence, at times signalizing a salutary and irreversible cooperation between South-American countries and at others, pointing out to divergencies of insuperable roots - at least in short and medium terms, and a role for Brazil that is still uncertain in such process. 


\section{THE "SOUTH-AMERICAN SPRING"}

In response to the socio-economical deterioration of the 1990s, which dragged tens of millions of people to poverty in a region already characterized by great amounts of different kinds of inequalities - consequence of the almost-unconditional adherence to the precepts of the "Washington Consensus" -, added to an extremely complex and fragile political framework characterized by a gradual corrosion of institutions, the turning of the millenium signalized to a new dawn in which, differently from the previous scene, new governments, increasingly identified with the citizenship and social movements demands, experimented an oportunity in the conduction of national business.

Symbolized by Hugo Chavez' rise to power in 1999 in Venezuela, this kind of "South-American spring" would be followed by the rise of new leftist and centre-leftist governers along the first decade of the new century, such as Luiza Inácio Lula da Silva in Brazil (2003), Néstor Kirchner in Argentina (2003), Tabaré Vázquez in Uruguay (2005), Evo Morales in Bolivia (2006), Rafael Correa in Ecuador (2007) and Fernando Lugo in Paraguay (2008), Who opened doors - supposedly - to other possibilities and forms of cooperation in this truly new context.

The great contradiction is that, in contrary to what common sense appears to suggest, the emergency of these new liderships, followed by intense popular mobilization and its entourage of previously excluded from the political process, ended up taking us to a new situation, in which,

The social basis of these new liderships tend to consider that a fair amount of its social and economic struggles are consequences of the impacts that come from outside, and therefore demand from their rulers a more autonomous international positioning. So they won't lose their domestic political support, these leaderships sometimes exacerbate their speeches and practices in their foreign policy. The consequence is that, paradoxically, the new SouthAmerican wave of "leftist" leaders, which is supposed to facilitate a future foreign policy agenda of regional integration by proximity of ideological identities, ends up operating against this tendency. ${ }^{1}$

There weren't few ocasion where disputes in the subregional level, either by intervention of the Andean Community of Nations (CAN), South- 
ern Common Market (Mercosur) or even the Bolivarian Alliance for the Americas (ALBA), dissipated the energy that could be channelled together and explicitated the heavy backstage quarrels that orient national chancelleries. ${ }^{2}$ Therefore, the necessary epistemological leap from governability to governance would be once again overshadowed.

Nonetheless, the historical hypothesis of war and conflict were already subjects of the past. The development of oriented preparation regarding the function of capacities (not to be confused with capabilities) instead of old threads, is increasingly becoming a reality. The end of cold war, the new democratic post-authoritarian regimes environment, the intensification os the globalization process and what has been conventioned to be called "new threats" (normally via non-state actors) has exponentially increased the adoption of measures of mutual trust between South-American states.

On the other side, the old antipathy that many of these new presidents from the subcontinent nurtured for Washington, combined to the low-skilled way with which the North-American interests were conducted in here, ended up showing the increasing feebleness of institutions and mechanisms such as the OAS, the Inter-American Defense Board (IADB), the Inter-American Treaty of Reciprocal Assistance (TIAR), amongst others, giving out unmistakable signs of depletion of the hemisphere's security system structured since the 1930s and 1940s.

The recent movement of the securitization of the terrorism phenomena in the North-American political agenda ended up creating contradictory answers in South America, when not open confrontation. The low strategic priority historically given from the yankee Department of State to the region, ${ }^{3}$ combined to the profound change in the international agenda of this country after setember of 2001, the repeating setbacks in themes such as the adoption of the Free Trade Area of the Americas (FTAA), the failures regarding the election of their own candidates secretary-general of OAS, the negative responses from Chile and Mexico (at the time, rotative members os the UN Security Council) in giving their support on the invasion of Iraq in 2003, amongst other questions, ended up consolidating na imaginary of geographic space where it was possible and susceptible to develop more independent-like policies, resulted from a wider margin of authonomy. Furthermore, the exit of hibernation state in which the russian bear has stayed during the 1990s and the expectacular chinese growth and its arrival in the subcontinent have helped to make this framework 
even more complex.

However, the fact to which we are here trying to bring attention on is that, despite the (punctual or not) quarrels between different South-American states in the processo of construction and conduction of regional integration, it looks like as though there is a common thread (except for Colombia) regarding the theme of security and defense, which is the aspiration of the political leaderships for the end of North-American heteronomy and interference in the region. In this particular case, South America concretely looks like it is living a new reality, with even the blooming of new institutionalities, such as the South-American Council of Defense and other mechanisms, regardless of a worrying approximation between Paraguay and the United States, particularly after the suspension of that country in Mercosur and UNASUR in 2012. ${ }^{4}$

However, despite the important transformations that took place in the South-American reality, this new framework did not represent per se the breakup or renounce of the "small sovereignties" imperative logic, much necessary to the creation of transnational arrangements that repeatedly appear as a desire in the speeches of numerous local leaders and that, it is worth remembering, are sought at least since the Congress of Panama, in 1826, idealized by Simon Bolivar. The different and sometimes conflicting visions about what is integration and how it should occur (in the most varied fields) continue do predominate.

\section{BRAZIL IN THE NEW CONTEXT}

President Luiz Inácio Lula da Silva’s inauguration in office in January 2003 would bring important changes not only on a domestic level but also externally, given the major regional player status and also Brazil's physical, political, economic and military dimensions. ${ }^{5}$ We should recall that a stronger assertiveness regarding South-American cooperation and integration, even though not completely new when we look back at History, gains a brand-new shape from the effort - in a Brazilian perspective - initiated with president Fernando Henrique Cardoso, deepened by president Lula da Silva (both rulers backed by a strong presidential diplomacy) and again renovated by present president Dilma Rousseff.

In this century, beyond physical and economical integration sought, Brazil has worked in South America trying to encourage a common security agenda and counter-arrest North-American influence in the region 
(originally centered in the expansion of military bases in the subcontinent, reactivation of the US Fourth Fleet and fight against terrorism and drugs). The agreement on such common agenda would allow, theoretically, even the creation of a security community, given the territoriality's centrality in the dynamics of security studies. However, regional or subregional level likewise, obstacles have appeared.

The integration march has proceeded with advances, deadlocks and sometimes drawbacks, since the First South-American Summit, which took place in August/September 2000 in Brasilia, with the region's heads of state. If geography is a destiny, as geopolitics would put it, Brazil and its neighbours seem to be awakening from a long period of lethargy and inaction, aware of the need of agreement on a common agenda of opportunities and challenges. As a mean to escape the traps sometimes placed by fragile institutionalization of democracy and the region's institutions, populist ravishments and/or disputes for leadership in the South-American integration process, Brazil opted for a bet on a kind of blend, since Cardoso, maintaining the previous open regionalism, based on physical integration (through the Initiative for the Integration of the Regional Infrastructure of South America/IIRSA), aiming for the promotion of the development of infrastructure for transportation, energy and communications. ${ }^{6}$ Highlighting the progress of Brazilian perspective, we could now list as priorities the structuring of the South-American Community of Nations/ CASA (later renamed Union of South-American Nations/USAN), the creation of the Council of South-American Defense (in open opposition to the US, which tried to use Colombia to block this Brazilian initiative) and the admission of Venezuela as a full member of Mercosur (Bolivia has already signed the adhesion protocol and negotiations with Ecuador have advanced positively).

The Council of South-American Defense is the boldest proposal within the theme hereby discussed, in which it appears not to be necessary to explicitate defense against who or what. ${ }^{7}$ It is important to point out that between the presentation of the proposal, initiated with backstage bilateral talks throughout 2006-2007, and the public exposition and aproval of the CSD in December 2008, many were the articulations to overcome the differences of shape and content between its 12 member, and also to bury the idea that such instrument intended to consolidate Brazilian hegemony in the region. ${ }^{8}$ Between a Colombia that saw itself isolated, enclosed by leftist governments (not to mention the narrow political-ideological bonds 
with Washington and colaboration in the military area via Plan Colombia), and an histrionic Venezuela that defended the creation of a common operational military tool called South Atlantic Treaty Organization (SATO, inspired on the North Atlantic Treaty Organization/NATO), ${ }^{9}$ the proposal that ended up chosen was the one regarding the implantation of a softened structure - subordinate to a regional multipurpose block such as USAN -, of harmonization between its members, able to encourage exchange in the fields of security and defense, in which decisions are only valid if agreed upon by consensus and with the prevision of at least one annual summit between its ministers of Defense. ${ }^{10}$

For some of the actors in this process, in speeding up the conformation of this arrangement (particularly from a Brazilian perspective), there were: 1) the possibility of a spill-over of "new threats" to other countries, such as Colombian narcotrafficking to Brazil and a quarrel involving Colombia, Ecuador and Venezuela for the death of Raúl Reyes (number two in the hierararchy of the Revolutionary Armed Forces of Colombia - People's Army/FARC-EP); 2) the subregional conflicts such as disputes involving Peru and Ecuador, Bolivia and Chile, amongst others; 3) the authocthon movements spreading a separatist feeling, as seen in Bolivia and Paraguay; 4) to stop bolivarian impetus in the region, especially through the isolation of Venezuela and/or other members of ALBA; 5) to avoid an arms race in the region, with the growing adoption of mutual trust measures (Confidence Building Measures/CBMs); 6) to set the South Atlantic as a peaceful area, vital and free of nuclear weapons, and one of power projection to Brasil, not allowing the presence of extra-regional powers (such as the UK and Falklands/Malvinas issue); 7) to block the materialization of a strategic policy for hemispheric security definied by the United States for the subcontinent; and, last but not least, 8) to accomplish Brazilian Foreign Policy strategic goals of consolidating a South-American integration process. ${ }^{11}$

Effectively, more than ever, stability plays a fundamental role for the acceleration of Brazil's development and consolidation of its global player intention, not only for the blooming of its potentialities but equally for showing the world its performance as provider of an important regional stability. ${ }^{12}$ Since 2004, the United Nations Stabilization Mission in Haiti (MINUSTAH)'s command has no other reason beyond protocol justification of humanitarian help, etc., consisting in a true turning point in the Brazilian modus operandi on UN peacekeeping operations. ${ }^{13}$ 
At the same time, as Alsina Jr. (2009, 181) points out, "the territorial satisfaction and the non-securitization of threats originated in neighbour countries allow the country [Brazil] to prioritize the development's dimension instead of the strategic-military dimension". However, if it has been like that historically, a country with over 15,000 kilometers of dry borders, approximately 8,000 kilometers of seashore and a huge aerial space, ${ }^{14}$ which reivindicates a permanent seat in a possible reconfiguration of the UN Security Council and intends on being guarantor of concordance in its surroundings, cannot do without a credible military structure and neglect its means of defense.

Under numerous circumstances, Brazil hasn't really succeeded, to say the least, in building a coherent and credible speech towards its regional partners, a consequence of a blurred identity profile. When talking about the subcontinent and Brazil's relationship towards it, while president FHC said that "we can only succeed if we are capable of being other countries' comrades. We cannot suffocate, take unnecessary advantage. Advantave is strategic, it's not due for tomorrow", sometimes there are statements such as president Lula's saying that "it is impressive how all these countries are almost demanding Brazil to lead South America” ${ }^{15}$ Regarding Lula da Silva's efforts towards the materialization of regional integration and the action BNDES (Brazilian Economic and Social Development Bank) started to play to set IIRSA as one of the building blocks of Brazilian foreign policy, there is still a general understanding of Brazil as a hegemon, giant and subimperialist, penetrating hearts and minds of most of its South-American neighbours. ${ }^{16}$ For such perception to vanish, it is vital to advance, effectively reducing import assymetries that still characterize the countries in the region and betting for real on regional integration (not only as a safeguard tool agains common external threats of different natures and/or a kind of soft balancing). Not only smaller neighbout but also Brazil would be a great beneficiary if such process took place, definitely dissipating the image of a "selfish giant" - as Andres Oppenheimer nicely put.

President Dilma's struggle resides in a resilient external framework of crisis, which directly impacts the domestic, regional and global spheres (limiting the Brazilian state's capacity of action in the international system), combined with the feeble enthusiasm with which the ruler handles international issues. On the other hand, the growing probability of Argentina's near convulsion, added to an economic and political fragilized 
Venezuela, show themselves as a chance for Brazil to turn this crisis into an opportunity.

Therefore, the construction of an active and proud security community aimed at regional stability appears itself as a central theme and task on the broader boudaries of this process, considering that, even with Brazilian participation trying to reduce suspicion, create undestanding and facilite conversation, the so-desired South-American identity in terms of defense is, as of this moment, still a promise unfulfilled. However, policies in favor of integration advance in two different speeds, sometimes faster in the political field - like the Council of South American Defense -, notwithstanding, followed by an incompatible rhythm in the economic field and others, and also a more solid institutional architecture, which is able to support a deeper and more profitable network integration.

Still: Brazil needs to lead this process without loss in its strategic partnerships, leaving the exercise of a timid leadersip and regulating winnings - being it desirable that this happens in an associated way. We believe that it is the moment to overcome the sterile debate regarding the Brazilian vision over South-American integration - if it is an end in itself or a platform for its global insertion, since there is no incompatibility, but a complementarity between these two possible ways in the reducing of fragilites and maximization of synergies. The time is long gone for overcoming hesitations, embarrassements and perplexities from these last years, which, in fact, have only terrified and paralised many of the initiatives towards integration.

\section{THE DIFFICULTIES IN THE CONSTRUCTION OF A NEW STRATEGIC CULTURE}

The construction of a new strategic culture, despite the political approximation and increase of intra-regional commerce that followed the end of military regimes in the region and the end of cold war, is not one of the easiest tasks. It consisted in centuries of the neighbours noticing each other as their main threats and guiding the preparation of their Armed Forces towards war with the countries in their surroundings.

Previous considerations and discussion on a new stretegic culture necessarily take us to a few questions: was there an important shift in the global distribution in the international system in the last few years? How did this partition of power affects the South-American options, particu- 
larly the Brazilian ones? Do we have strong regional institutions? Does the civil/political world rules the arms/military world? These and other issues made a central impact on the thought we are here building.

The first element to be highlighted is the relative change in the United States position in the international system. The September 11, 2001 attacks, the first major-proportion ones in the North-American continental portion since its rise to the condition of power not only shook the contemporary feeling of being unscathed by the part of the population which launched its political system in an international cruzade called war on terror. A financial crisis started in 2008, whose epicenter took place in Said country equally implied in a - although relative - decline of the superpower in the set of social interactions that developed themselves in the international sphere. Even so, without getting into details of the Russian rebirth and growing Chinese protagonism, we believe that the international system's polarity hasn't been altered in the post-Cold War period, ${ }^{17}$ despite new vertexes of power that have emerged in the present century, but hasn't been able to break unipolarity.

Besides, the North-American strategic and military urgencies - notably Afhganistan and Iraq - enabled that, in a region that even in the Cold War period has never been on top of the great power priorities, a new division of responsibilities took place, in which Brazil rises as guarantee of South-American stability (including trying to moderate Bolivarian countries), in a kind of leadership of consent without, however, severely confronting Washington on a global level.

As a background, since the foundation of Mercosur in 1991 until the establishment of USAN in 2008, we have seen a swarm of instances, processes and institution that must be strengthened, matured and wellgrounded. The rapid and constant modifications and regressions of the points previously agreed upon between the subcontinent countries little contribute to the strengthening of regional institutions - necessary condition, however insuficient - and support for the deepening of interstate security relations, which is the point here that interest us the most. The most dramatic example may be Mercosur, proposal of a Common Market, currently at the stage of Customs Union (although imperfect!), which has been taking important sectors of the block's different constituent states to defend that this arrangement advances towards a Free Trade Area. The reflexes of such instability may be felt, e.g., through the non-materialization of a Security and Defense Agency for Mercosur as expected for more 
than a century and whose headquarters would be in Montevideo (as an example such as Parlasur), making it more difficult the passage and advance on the bilateral dialogue level the the subregional dialogue one. ${ }^{18}$

Thus, in the beginning of the new century's second half,

The integration processes remain, but without deepenings. In the area of defense and security, a significant level was reached of mutual trust mechanisms, which seem satisfactory to the countries. That it, new and bold steps are not established, even with the creation of USAN and the Council of South-American Defense. ${ }^{19}$

In addition, without frequently desregarding the civil police's ineptitude and the slowness/mistrust of national bureaucracies, it is worth pointing out that actors in uniforms are not mere supporters, and such a deep-rooted conservative mentality as the military does not alter through a simple political regime change and a new historical reality. It requirs, in fact, a deep transformation in the cultural field and socially constructed signs. In this direction,

Cultural transformations are naturally large processes, not deprived from marches and countermarches. The changes in the conceptions of security are partly derived from these processes, and in the region have been marked by deep debates regarding what security means, what is the role of the military forces and fundamentally which are the views that each country has on what defies its security scenario. This causes impact on the multilateral possibilities. ${ }^{20}$

Simultaneously, the civil democratic control over militaries should be a consolidated fact. So, political directin should point out to which Armed Forces they are ambitioned considering the strategic profile intended by the country, which should contemplate the resizing of its means, which include personal, material, educational, etc. We would like to signal that, in some countries of the region, the military apparatus comes dangerously assuming tasks that are cognisable to the civils, with not at all auspicious possible outcomes, beyond the already traditional maintenance of guarantor's mission (in different levels) of the political and institutional order that it preserves in many of these nations.

A new strategic culture does not arise between twilight and dawn. In a summarized way and thinking in terms of regions and body of know- 
lege on the theme, it is worth listing the creation of mechanisms such as the "American Treaty on Pacific Settlement"/Pact of Bogota (1948), the "Latin America Nuclear Weapons Free Zone Treaty"/Treaty of Tlatelolco (1967), the "South Atlantic Peace and Cooperation Zone" (1986), the "Mendoza Agreement" between Argentina, Brazil and Chile (1991), prohibiting the proliferation, possession and use of chemical and biological weapons, the "South-American Peace Zone"/ Brasilia Communiqué (2000), the inauguration of the "South America Defense Ministers Meeting" (first of them taking place in Rio de Janeiro in 2003), besides the growing relations of the bilateral relations between Brazil and Argentina since de 1980 s in the nuclear Field, with the abdication of atomic weapons fabrication and use by both countries and the institution of the BrazilianArgentine Agency for Accounting and Controlo f Nuclear Materials / ABACC (only binational organization of nuclear safeguards in the world), culminating on the "Bilateral Agreement on Cooperation in Matter of Defense", signed in 2007. ${ }^{21}$ At last, na expressive heap and a not-despicable tradition of trying to easing the tension in the regional security and defense environment.

Since 2009, with the effective start of its activities, the Council of South-American Defense has appeared as an important concert space where, despite regional/ideological idiosyncrasies between CAN, Mercosur or even ALBA, import advances have been registered, such as the definition of common Plans of Action on the security and defense theme, the stablishment of mutual trust measures, the creation of the Centre for Strategic and Defense Studies (CEED) in Buenos Aires, the construction of a common methodology for measuring the expenditures in defense, an important exchange on the matter or military formation and capacitation, the recently announced South-American Defense School, amonst other equally meritorious points. However, challenges remain.

\section{FINAL CONSIDERATIONS}

Considering what has been said, the regionalization of the security and defense architecture in the subcontinent continues, but with new questions: what have we been watching, arms race or refit? And have the historical border contentious and territorial quarrels been overcome? Are the national spaces and identities respected mutually? How to avoid unbalances where the economy/GDP - as well as the expenditures in the 
area of defense - only in Brazil represent more than half of all the other countries' of the subcontinent combined? We can - definitely - talk about overcoming of the dicontomy Pacific (instability arch/CAN) versus Atlantic (instability arch/Merocsur)? How does the amazonic issue presents itself nowadays? The risk of balcanization of the region's countries has passed? Has the North-American strategic tutelage been contained?

In fact, the Council of South-American Defense has not yet passed through any acid test that able to put in check the efficacy and efficiency of this institutional structure, beyond the (important) crisis management and generation of positive externalities on the field of security and defense. Simultaneously, the so-called negative agenda, which characterized the USA-South America relationship in the post-Cold War period, centered on the fight against drug trafficking, terrorism and other crimes of police nature, was partially overcome. Despite all of this, the Regional Security Subcomplexes of the Southern Cone and Andean North still live different very dynamics, which call for better articulation and organization. The South-American integration and structuration of a centered Regional Security Complex have advanced in a non-negligible, frequently supported by fragile bases as a source of nationalist rhetoric, defense of sovereignty and non-intervention. ${ }^{22}$

Brazil's behaviour, main guarantor of the creation of CSD, lacks coherence and presents itself numerous times in a diachronic way. If, in one hand, on the highest political level, Brasilia works for the materialization of this institutional arrangement (which already represents $2 / 3$ of its exportations in arms); on the other hand, it lacks the initiative to engage the Ministry of Defense and, particularly the Brazilian militaries, to act in a more decisive and dedicated way for the materialization of this important initiative and its operative mechanisms. In practice, many high officers and officer generals see this business aprehensively, not rarely classifying it as an "extravagance", result of daydreams from a leftist government that would not find correspondance in reality.

If, in desillusion, regional integration has worked as a strategy to gain more voice (and strength) to the region's pleas in a world of growing power assymetries, this cannot eclipse the absolute fragility that such instrumentalization of this important process represents to a more systemic cooperation, notwithstanding the punctual advances. The passage from collective security to cooperative security is a fact. At last, the seed has been planted but for it to grow, attention, care and dedication will be 
needed. The feeling cultivated by parto of the Brazilian elite regarding its country as a "sweetheart" in the region is feeble in its origins. If Brazil wants indeed to lead, it takes will and mobilization for such a task and may it be aware that this comes with a price (and not a cheap one!)

Throughout the first decade of the 21 st century, the meetings not only increased in periodicity but also new institutional arrangements were thought and implemented. As a final result, we verified in this brief dynamics balance advances and challenges in the fields of security and defense in the South-American geographic space, in which the current processo $f$ integration is marked by numerous ambivalences, inconsistencies, when not open opositions. Plus: the construction of a new strategic culture, however admirable, will show itself to be of feeble foundation if not accompanied of respective deepening and creation of new institutionalities in the economic, social and cultural field, amongst others. So, this contribution, far from putting a final period on the debate, intends to bring up a reflection in an area that is still very little explored in the subcontinent, adding up to the other attempts of Brazil and South America contemporary interpretations and the roles they seek to play in the present century. 


\section{NOTES}

1. Dupas \& Oliveira (2008), 239.

2. Just to illustrate, this can be easily verified in the debates envolving the "biofuel diplomacy" (Lula) versus the "oil diplomacy" (Chávez).

3. Recently, Barack Obama's State Secretary John Kerry in a speech before the Commitee of Foreign Affairs of the House of Representatives classified Latin America as the United States " backyard". See "John Kerry, Secretary of State: 'Latin America is our back yard' " (http://english.pravda.ru/world/americas/2304-2013/124377-latam_backyard-0/; access in 04 may 2013) and Colombo \& Frechero (2012).

4. Before that, militart exercises from parto $f$ the Brazilian Army on the border with Paraguay at the emd of 2008 in a kind of informal revenge against "brasiguaios" (main agricultural producers of what country) Who suffered pression from local landless, mobilized na effective of thousands of men, bringing tension and discomfort to the région. To make it worse, since 2010 the guerrilla also turned into a new and sad reality in this suffering country, through the Paraguayan People's Army (EPP).

5. On the same year, it took place in Brazl for the first time ever the "First Meeting of South-America Ministries of Defese". Brazilian Foreign Policy throughout Lula's administration (2003-2010) is the stage for a loto f debate. Regarding security issues and the differential treatment such issues received during president Lula's two administrations, we suggest Villa e Viana (2010). 6. There are more thatn 500 projects that estimatively cost around US\$120 billions. For action, projects, planning áreas and IIRSA documents, see http:// www.iirsa.org/index.asp?CodIdioma=ESP (access in 15 mar. 2013).

7. For a chronology of the creation of CSD and the disputes envolving its institution, see Medeiros Filho (2010), Carvalho (2009) and Teixeira (2011). For a broader balance, cf. Pagliari (2009).

8. Na interesting panorama of how Brazilian insertion was thought since the 1950s until nowadays can be found in Vigevani e Ramanzini Júnior (2010). On this discussion regarding the Idea of Brazilian leadership, it is worth considering Danese (2009).

9. Before this oportunity, the last time that the creation of a similar mechanism was invented was back in the 1980s, in the beginning of Ronald Reagan's administration (1981-1989), in which Argentina and South Africa were 
enthusiasts of such North-American proposal. Brazil and other African countries - notably the ones from so-called Western Africa - sought to blcok such initiative. In the middle of 1982, however, with the beginning of the Falkland/Malvinas war, such daydream started to fade.

10. Regarding its functions and other atributions, see the website http://www. cdsunasur.org/ (access in 27 mar. 2013).

11. Personal notes from off the Record conversations with members of Itamaraty and Brazil's Minisitry of Defense.

12. For such, it is a fact that along with traditional soft power, there are numerous challenges for Brazil to increase its hard power. For example, na important limitation comes from the fact that this country is the only member of the denuclearizes BRIC block. Cf. Bertonha (2010) e Flores (2009-2010).

13. For details on Brazilian participation in Peacekeeping and Peacebuilding Operations, see Rezende (2012).

14. Suportung these elements, the reminder that many South-American nations are geographically smaller than many Brazilian federate state (e.g., if the Amazonas state was a country, it would be the third largest one in South America - after Brazil and Argentina), less populous (e.g., Minas Gerais state has a bigger population than Bolivia, Paraguay and Uruguay all together), of less economic weigh (e.g., if São Paulo state was a country, it would be the second biggest economy in the subcontinent, only behind Brazil itself), amongst other points, cannot avoid causing a bad feeling amongst its neighbours.

15. "Para América do Sul, liderança brasileira ainda é promessa" (http://www. bbc.co.uk/portuguese/reporterbbc/story/2008/03/080303_ams_abre1_diplomacia. shtml; access in 27 mar. 2013).

16. In a quick web search, this is easily found. To make it clear, see Le Monde Diplomatique Brasil. Dossiê Imperialismo Brasileiro. February 2009; “O imperialismo brasileiro preocupa a região", O Estado de S. Paulo, 23/10/2008; "El imperialismo brasileño seguirá intacto con el gobierno de Dilma", ABC Color (http://www.abc.com.py/articulos/el-imperialismo-brasileno-seguira-intacto-conel-gobierno-de-dilma-178977.html; access in 27 feb. 2013); Samuel de Jesus. “ 'Imperialismo Brasileiro': visões jornalísticas sobre a atuação brasileira na América do Sul e no mundo" (http://mundorama.net/2012/09/25/imperialismobrasileiro-visoes-jornalisticas-sobre-a-atuacao-brasileira-na-america-do-sul-e-nomundo-por-samuel-de-jesus/; access in 26 feb. 2013). For another point of view, cf. Curado (2011). 
17. In the middle of a "crisis", the United States of America, whcih concentrates $5 \%$ of world's population, in 2012 were still responsible for $43 \%$ of military and defense expeditures in the world. See http://www.sipri.org/databases/milex (acess in 12 jul. 2013).

18. Personal documents from when I worked at the Secretariat for Politics, Strategy and International Affairs in Brazil's Ministry of Defense, from 2003 to 2005.

19. Our highlight. Cf. Soares (2011), p. 104.

20. Our highligh. See Donadio (2011), p. 115.

21. To these, we could add Brazilian initiatives of Bilateral Agreements with Bolivia, Chile, Colombia, Ecuador, Paraguay, Peru and Uruguay, besides regional security Agreements signed by Mercosur with Bolivia, Chile, Colombia, Ecuador, Peru and Venezuela - all in this century.

22. For details, the relation between USAN and said subcomplexes was exposed in Fuccile and Rezende (2013) work. 


\section{RESUMO}

O século XXI inaugura um novo período no relacionamento entre os países da América do Sul e, em particular, do Brasil para com estes. A erosão da influência norte-americana na região (associada a outras prioridades), ao lado de um maior protagonismo brasileiro e um sistema internacional crescentemente multipolar com novos vértices de poder, afetaram decisivamente a forma pela qual a segurança e defesa no subcontinente eram pensadas e conduzidas há mais de meio século. Fundamentalmente, percebe-se um esforço que pretende representar uma passagem de um arranjo caduco de segurança coletiva em direção a um modelo mais dinâmico de segurança cooperativa, contudo de contornos ainda incertos e cujo resultado final segue em aberto.

Palavras-chave: América do Sul. Política de Defesa. Política Externa Brasileira. Integração Regional. Segurança e Defesa

\section{ABSTRACT}

The XXI century inaugurates a new period in the relationship between the countries of South America and, in particular, from Brazil to with these. The erosion of American influence in the region (associated with other priorities), alongside a greater Brazilian leadership and an increasingly multipolar international system with new vertices power decisively affect the way in which security and defense in the subcontinent were thought and conducted for over half a century. Fundamentally, we perceive an effort that seeks to represent a passage of a lapsed collective security arrangement towards a more dynamic model of cooperative security, yet still contours uncertain and the end result is still open.

Keywords: South American. Defense Policy. Brazilian Foreign Policy. Regional Integration. Security and Defense,

\section{REFERÊNCIAS}

$A B C$ Color. "El imperialismo brasileño seguirá intacto con el gobierno de Dilma" (http://www.abc.com.py/articulos/el-imperialismo-brasileno-seguira-intactocon-el-gobierno-de-dilma-1 78977.html; acesso em 27 fev. 2013). 
Alsina, J. P. S. "O poder militar como instrumento da política externa brasileira contemporânea”. Revista Brasileira de Política Internacional, 52 (2), pp. 173-191, 2009 .

Bertonha, J. F. "Brazil: an emerging military power? The problem of the use of force in Brazilian international relations in the 21 st century", 2010 (http://www. scielo.br/pdf/rbpi/v53n2/06.pdf; acesso em 18 jul. 2012).

British Broadcasting Corporation/BBC. "Para América do Sul, liderança brasileira ainda é promessa” (http://www.bbc.co.uk/portuguese/reporterbbc/ story/2008/03/080303_ams_abre1_diplomacia.shtml; acesso em 27 mar. 2013).

Carvalho, L. A. de. Conselho de Defesa Sul-Americano. In: Carvalho, L. A. de; Vera, C. G.; Peña, J. C. (Orgs.). Segurança e defesa na América Latina. Curitiba: Juruá, 2009.

Colombo, S.; Frechero, J. I. "Yes we can? A Política Externa de Obama para a América Latina: da decepção à autonomização da região". Contexto Internacional, (34) 1, pp. 189-222, 2012.

Conselho de Defesa Sul-Americano/CDS da Unasul. (http://www.cdsunasur.org/; acesso em 27 mar. 2013).

Curado, P. R. F. "O Brasil na América do Sul: sub-imperialismo ou liderança regional benigna?”, 2011 (http://rediu.org/ROCHA.mesa8.pdf; acesso em 27 fev. 2013).

Danese, S. A escola da liderança - ensaios sobre a política externa e a inserção internacional do Brasil. Rio de Janeiro: Record, 2009.

Donadio, M. Visões estratégicas na América do Sul. In: Oliveira, M. A. G. de (Org.). Comparando a Defesa Sul-Americana. Recife: Editora Universitária da UFPE, 2011.

Dupas, G.; Oliveira, M. F. de. A União Sul-Americana de Nações. In: Ayerbe, L. F. (Org.). Novas lideranças políticas e alternativas de governo na América do Sul. São Paulo: Editora UNESP; Programa San Tiago Dantas de Pós-Graduação em Relações Internacionais da UNESP, Unicamp e PUC-SP, 2008.

Fuccille, A.; Rezende, L. P. O Complexo Regional de Segurança na América do Sul: a Unasul e a concertação dos Subcomplexos Norte-Andino e do Cone Sul. In: OLIVEIRA, M. A. G. de (Org.). Cultura de Defesa Sul-Americana. Recife: Editora Universitária da UFPE, 2013. 
Iniciativa para a Integração da Infra-estrutura Regional Sul-Americana/IIRSA. (http://www.iirsa.org/index.asp?CodIdioma=ESP; acesso em 15 mar. 2013).

Jesus, S. de. “'Imperialismo Brasileiro’' visões jornalísticas sobre a atuação brasileira na América do Sul e no mundo”, 2012 (http://mundorama.net/2012/09/25/ imperialismo-brasileiro-visoes-jornalisticas-sobre-a-atuacao-brasileira-naamerica-do-sul-e-no-mundo-por-samuel-de-jesus/; acesso em 26 fev. 2013).

Le Monde Diplomatique Brasil. “Dossiê Imperialismo Brasileiro”. Fevereiro de 2009.

Medeiros Filh, O. "Conselho de Defesa Sul-Americano: Demandas e Agendas", 2010 (http://www.brasa.org/_sitemason/files/fLdvB6/Oscar\%20Medeiros\%20 Filho.pdf; acesso em 12 mai. 2011).

O Estado de S. Paulo. "O imperialismo brasileiro preocupa a região", 23 out. 2008. PAGLIARI, G. de C. O Brasil e a segurança na América do Sul. Curitiba: Juruá, 2009.

Pravda. "John Kerry, Secretary of State: 'Latin America is our back yard”"(http:// english.pravda.ru/world/americas/23-04-2013/124377-latam_backyard-o/; acesso em 04 mai. 2013).

Rezende, L. P. O Engajamento do Brasil nas Operações de Paz da ONU - Análise dos Efetivos Enviados e Recomendações para o Fortalecimento da Inserção Internacional Brasileira. Curitiba: Editora Appris, 2012.

Soares, S. A. Contendores apaziguados ou partícipes da cooperação? As percepções sobre ameaças e cooperação nas políticas de defesa de Argentina, Brasil e Chile na década de 1990. In: Oliveira, M. A. G. de (Org.). Comparando a Defesa SulAmericana. Recife: Editora Universitária da UFPE, 2011.

Stockholm International Peace Research Institute/SIPRI. (http://www.sipri. org/databases/milex; acesso em 12 jul. 2013).

Teixeira, A. O Conselho de Defesa Sul-Americano da Unasul. In: Oliveira, M. A. G. de (Org.). Comparando a Defesa Sul-Americana. Recife: Editora Universitária da UFPE, 2011.

Vigevani, T.; Ramanzini Júnior, H. "Pensamento Brasileiro e Integração Regional”. Contexto Internacional, (32) 2, pp. 437-487, 2010.

Villa, R. A. D.; Viana, M. T. “Security issues during Lula's administration: from the reactive to the assertive approach". Revista Brasileira de Política Internacional, 53 (special edition), pp. 91-114, 2010. 